\title{
An Interactive Deblurring Technique for Motion Blur
}

\author{
Yogesh K. Meghrajani \\ Department of Electronics \& Communication \\ Faculty of Technology, Dharmsinh Desai \\ University, Nadiad, Gujarat, India
}

\author{
Himanshu Mazumdar \\ Department of Electronics \& Communication \\ Faculty of Technology, Dharmsinh Desai \\ University, Nadiad, Gujarat, India
}

\begin{abstract}
An interactive deblurring technique to restore a motion blurred image is proposed in this paper. Segment based semiautomated restoration method is proposed using an error gradient descent iterative algorithm. In this approach, segments are automatically detected which are the best representatives of motion blur. Then the decimal parameters of the blur kernel are interactively derived; with extended precision using interpolation between pixels, with comparatively much lower error convergence rate. Once blur kernel is obtained, image is restored using Striling's interpolation formula. Experimental results show that proposed method gives sufficient restoration as interactive judgment gives the most desirable quality.
\end{abstract}

\section{General Terms}

Image Restoration, Image Processing

\section{Keywords}

Image Deblurring, Motion Blur, Image Interpolation

\section{INTRODUCTION}

One of the most common problems in digital image restoration is motion blur caused by an image captured from a moving platform especially at low light condition having long exposure time of camera. However, capturing well focused photos may not be guaranteed as most camera modules are not robust at low light condition for camera shake. Image deblurring is the process of obtaining the latent (unblurred) image by using the knowledge of the degradation factors. Recovering a deblurred image from a single, motion-blurred photograph has long been a fundamental research problem in digital imaging. In [1], Ko et al. discuss about different forms of hardware and software approach from which they conclude that software approaches such as post-processing are more appropriate for embedded system. They propose blur detection and estimation method considering uniform motion blur. Here, detection is constructed by the Bayes discriminant function with the statistics of the gradients of the input image and estimation of the blur kernel is by extracting the main ripple components from the log spectrum of the blurred image. [2] proposes variational Bayesian theory to deblur the camera-shake blurred image. To remove motion blur from a single image, [3] uses statistics of derivative filters in images to model derivative distributions as a function of the width of the blur kernel. A space-adaptive regularization method for image restoration is extended in [4]. [5] computes a deblurred image using a unified probabilistic model of both blur kernel estimation and unblurred image restoration. [6] proposes motion deblurring algorithm that exploits sparsity constraints of image patches using one single frame. This method iteratively utilizes sparsity constraints to recover latent image, estimates the deblur kernel, and updates the dictionary directly from a single image. A patch based image degradation model [7] is proposed for estimating the sample covariance matrix of the gradient domain natural image. The
PSF is computed using phase retrieval technique to remove the ambiguity introduced by the absence of the phase. [8] discusses methods for estimating linear motion blur. The angle of motion blur is estimated using cepstrum, Gaussian filter, and Radon transform. The extent of the motion blur is estimated using two different cepstral methods. Concepts of artificial intelligence are applied [9] for restoration problem in which images are degraded by a blur function and corrupted by random noise. This methodology adopted back propagation network with gradient decent rule which consists of three layers. Neural network based multi-valued neurons is used for the blur and blur parameters identification [10]. The results of this identification are used as the main parameters for the image restoration using the Wiener, Tikhnov or inverse filters.

Deblurring technique comprises of blur kernel estimation and restoration of image using estimated kernel. Estimation of blur kernel is conventionally performed statistically or deterministically. In case of statistical approach, methods use prior statistics learned from a large set of images $[2,3,5]$. Deterministic techniques are often developed using only synthetic images $[6,8,9,10]$ which may lead to incorrect blur kernel. If incorrect blur kernel is chosen [8], the image will be rather distorted much more than restored. Often in the real world scenario, it is required to restore an important image irrespective of the cost and speed of operation. Few examples are identifying alphanumerical content of a number plate, recognizing face or object from a blurred image captured for criminal detection, astronomy, and remote sensing. In such circumstances, deblurring score depends on the preconceived knowledge of the object shape. Context sensitive knowledge of human being is the best resource to interpret the latent image with sufficient quality, i.e. differentiation between letter ' $\mathrm{S}$ ' and number ' 5 '. For such important images, interactive improvement in quality could be the best performed by human perception. In certain applications, source of blurring is common for large number of image frames, i.e. satellite imaging [11] or streaming video from a moving platform. Detecting interactively, blur kernel will cause a smaller overhead of manual processing with higher accuracy in kernel parameters by virtue of human object validation skill.

In this paper, we propose an interactive deblur method to iteratively restore decimal parameters of blur kernel using image interpolation from a single blurred image. Interpolation is employed to find sub-pixel intensity value of entire image, to restore the blurred image for decimal parameters of blur kernel. Utilization of interpolation is more rational for low resolution images where blur parameters are not analogous with corresponding integer values. The effect will be more prominent while deblurring an image containing alphanumeric content. The best locations to examine blurring are the places having high gradient or edges of the well known object. To increase speed of operation, interactive window of small segments; having highest gradient in horizontal and vertical direction, is used for quick visual feedback. Our algorithm 
automatically identifies such segments and performs deblurring with initial nominal arbitrary blur parameters. An error gradient descent iterative method is used to optimize blur kernel on a smaller set at much higher speed. Result of each of the iteration is evaluated by human perception. Once blur kernel is obtained, global update is made. Experimental results are shown for synthetic motion blurred and unknown motion blurred images.

\section{PROPOSED METHOD}

A linear, position invariant degraded image is represented in spatial domain as,

$g(x, y)=f(x, y) * h(x, y)+\eta(x, y)$

Where, $f(x, y), h(x, y), g(x, y)$, and $\eta(x, y)$ represent latent image, degradation function, observed image, and additive noise respectively. The "*" in (1) symbolizes for 2-D convolution given by [12],

$$
\begin{gathered}
f(x, y) * h(x, y)=\sum^{M-1} \quad \sum^{N-1}(m, n) h(x-m, y-n) \\
m=0 \quad n=0
\end{gathered}
$$

Where, $\mathrm{M}$ and $\mathrm{N}$ represent image width and height respectively.

The objective of restoration is to obtain an estimate, $\hat{f}(x, y)$ of the latent image.

Accurate estimation of blur kernel mostly involves real value of offset and direction. Presently, low cost mobile phone cameras and webcams use low resolution imaging. In low resolution image, blur due to smaller offset is equivalent to larger offset in high resolution image. Hence, a decimal arithmetic is more appropriate in low resolution image for deblurring. As a result, restoration needs real coordinate system.

\subsection{Image Blurring and Deblurring Model}

Image motion blurring process can be considered as a spreading of image by real value of blur kernel and the interacting with other pixels. Suppose an image $f(x, y)$ undergoes planar motion and $\mathrm{x}_{0}(\mathrm{t})$ and $\mathrm{y}_{0}(\mathrm{t})$ are the time varying components of motion in the $\mathrm{x}$ and $\mathrm{y}$ directions, respectively. The total exposure at any point of the recording medium is obtained by integrating the instantaneous exposure over the time interval during which the imaging system shutter is open.

For a linear distribution of intensity in a small segment of blurring width, we find the continuous and discrete integration is approximately same. In this model, we assume blur length is small compared to image dimensions and pixel values are approximately linear within blur length. In case of linear motion blur without $\eta$, to represent a blur image we first translate original image using affine transform [13] as,

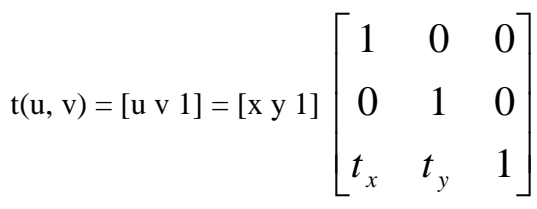

Where, $\mathrm{t}_{\mathrm{x}}=\mathrm{L} \cos \theta$;

$$
\mathrm{t}_{\mathrm{y}}=\mathrm{L} \sin \theta
$$

Here, $(\mathrm{x}, \mathrm{y})$ are pixel coordinates in the original image, (u, v) are pixel coordinates in the translated image, $\mathrm{L}$ is the blur length, $\theta$ is the blur angle of the blur kernel.

For real world images range of $\theta$ is $0^{\circ}$ to $360^{\circ}$ and $\mathrm{L}$ is any real value which makes $t_{x}$ and $t_{y}$ real value. Depending upon values of blur kernel $L$ and $\theta$, blur direction changes. As $t_{x}$ and $t_{\mathrm{y}}$ have real values, sub-pixel level translation is required. To translate an image for real values, interpolation is required to estimate sub-pixel value.

Here, we use central difference interpolation using Stirling's formula. Stirling's interpolation uses eight neighboring points compared to bicubic interpolation which uses 16 neighboring points with required accuracy and less computational burden. $\mathrm{L}$ and $\theta$ are selected interactively which changes $t_{x}$ and $t_{y}$. In this model, any value of step size can be selected for blur length and angle.

Pixel-wise average of the translated image and original image gives a blurred image as;

$g(i, j)= \begin{cases}\frac{[f(i, j)+t(i, j)]}{2} & \text { if } 0 \leq\left(i+t_{x}\right)<M, \text { and } 0 \leq\left(j+t_{y}\right)<N \\ f(i, j) & \text { Otherwise }\end{cases}$

Where $f(i, j)$ is original image, $t(i, j)$ is translated image, $g(i, j)$ is blurred image, $\mathrm{i}=0$ to $\mathrm{M}-1, \mathrm{j}=0$ to $\mathrm{N}-1, \mathrm{M}$ is width, and $\mathrm{N}$ is height of an image. Depending upon the blur direction $t_{x}$ and $t_{\mathrm{y}}$ will have positive or negative real values.

For a given blurred image, reverse process is pursued. Latent image $f(x, y)$ is obtained by,

$f(x, y)= \begin{cases}{[2 * g(x, y)]-t_{B}(x, y)} & \text { if } 0 \leq\left(x+t_{x}\right)<M, \text { and } 0 \leq\left(y+t_{y}\right)<N \\ g(x, y) & \text { Otherwise }\end{cases}$

Where $\mathrm{g}(\mathrm{x}, \mathrm{y})$ is blurred image, $\mathrm{t}_{\mathrm{B}}(\mathrm{x}, \mathrm{y})$ is translated blurred image, $f(x, y)$ is latent image, $x=0$ to $M-1, y=0$ to $N-1, M$ is width, and $\mathrm{N}$ is height of an image.

Depending on the values of $t_{x}$ and $t_{y}$, initial pixels are available in latent image. Using these pixel values, $t_{B}$ is generated by iteratively interpolating the formulating latent image by decimal parameters of blur kernel.

\subsection{Image Interpolation}

To translate entire image for decimal parameters of blur kernel, sub-pixel value is required to be found out for the entire image. As sub-pixel value is not available for digital image, to estimate its value, 2D pseudo-cubic interpolation technique is applied as shown in fig. 1 .

Interactive deblurring method is based on Stirling interpolation formula [14]. Stirling interpolation uses central difference for estimating the slope of an arbitrary nonlinear function with the set of sampled points. For interactively selected blur kernel, ceiling value is considered as a central sample and its value is estimated using central difference. To get accurate value, central difference table is prepared up to fourth order difference in each direction. Here, $x_{1}, x_{2}, x_{3}, \ldots$. , $\mathrm{x}_{\mathrm{n}}$ are pixels in $\mathrm{X}$-axis (horizontal) direction. Each horizontal pixel has value as $X_{1}, X_{2}, X_{3}, \ldots, X_{n}$. The fourth order interpolation formula is [14], 
$X=X_{0}+p \mu \delta X_{0}+\frac{p^{2}}{2 !} \delta^{2} X_{0}+\frac{p\left(p^{2}-1\right)}{3 !} \mu \delta^{3} X_{0}+\frac{p^{2}\left(p^{2}-1\right)}{4 !} \delta^{4} X_{0}$

Where, $X$ is model, $X_{0}$ is central pixel value, $\mu$ is the averaging operator, $\delta$ is the central difference notation, $\mathrm{x}=\mathrm{x}_{0}$ $+\mathrm{ph} ; \mathrm{h}$ is difference between two pixels, which is 1 in this case.

Similar procedure is followed for pixels in $\mathrm{Y}$-axis (vertical) direction. For color image, procedure is required to be pursued for each color component. For above studies, boundaries of two pixels on all sides are ignored. Entire procedure is repeated for different values of motion kernel. Every time image is visually observed and the best appeared image from human perception point of view is considered as a final result.

\subsection{Image Segment based Restoration}

To speed up restoration process, a small segment of the image representing the highest gradient is selected as an anchor point. Experimentally, it was found that we require minimum $64 \times 64$ pixels segment size to visually validate an image segment. Firstly, normalized Sobel operator [15] is applied on the grayscale version of the target image. Summation of grayscale intensity of pixels is performed on randomly selected small image segment of the resultant image. For blur image, this summation will be high at the highest gradient. Such process is iteratively performed at different random segments of an image for random search of the highest gradient segment.

To verify the authenticity of this algorithm, Gaussian image of different grayscale intensities as shown in fig. 2 (a) is generated using Gaussian function,

$$
\frac{-K *\left(x^{2}+y^{2}\right)}{2 * \text { Variance }}
$$

Where, $\operatorname{Variance}(\mathrm{X})=\mathrm{E}\left[\mathrm{X}^{2}\right]-\mathrm{E}[\mathrm{X}]^{2}$

$\mathrm{E}\left[\mathrm{X}^{2}\right]=$ mean of the square; $\mathrm{E}[\mathrm{X}]^{2}=$ square of the mean, $\mathrm{K}=$ constant, $\mathrm{w}, \mathrm{h}=$ width and height of an image respectively, $\mathrm{x}$ varies from $-\frac{\mathrm{W}}{2}$ to $+\frac{\mathrm{W}}{2}$ and $\mathrm{y}$ varies from $-\frac{\mathrm{h}}{2}$ to $+\frac{\mathrm{h}}{2}$

We have applied our algorithm on this image. As blur and gradient are vectors, experimentally we found that if these two vectors are orthogonal, blurring could be zero even though processed area has the highest gradient as shown in fig. 2 (b). To overcome this limitation of the algorithm, we modified it to two segments highest gradient algorithm in which we have selected two segments. These two segments are selected as per the highest gradient in horizontal (Gx) and vertical (Gy) directions of the normalized Sobel operator output. Aim behind selecting this approach is if blur has maximum value in vertical segment, it will have minimum value in horizontal segment and vice-versa. So, this will increase reliability of the system. Fig. 2 (c) - (e) shows experimental results of the above algorithm.

\section{EXPERIMENTAL RESULTS}

To validate the image deblur concept, motion image deblur simulator is developed. Motion blur is achieved by averaging the image and the same image translated by decimal parameters of blur kernel. Image is reformed by applying blur kernel obtained from simulator. Preliminary restoration direction changes as per value of blur kernel. We also carry out experiments with images blurred by randomly generated blur kernels. Experimental result in fig. 3(b) shows blurred image of fig. 3(a), affected by decimal blur kernel and fig. 3 (c) - (e) show recovery with integer kernels and decimal kernel respectively. Results of fig. 3 (c) - (e) exhibit the significance of interpolation for low resolution image.

Further, we evaluate our method for alphanumeric image, for which we utilize blurred image of fig. 4 (a), obtained from paper [1]. In fig. 4 (b), we show result of the Ko. et al. [2009] in which blur is effectively present on the left side, and heading. Fig. 4 (c) shows our method is able to recover image with fewer ringing effects and exhibits clearer visual appearance.

At last, we present restoration of blurred image with camera shake blur, captured from mobile phone camera having spatial resolution of 2 megapixel. Fig. 5 (a) shows blurred image and fig. 5 (b) shows restored image. Resultant number plate can facilitate human being to identify original number "GJ-1-BP2133 " of number plate. Ringing artifacts are mainly due to unknown nonlinear camera response [16] from mobile phone which is outside the scope of this paper as blurring is considered as linear process.

\section{CONCLUSION}

Blind deconvolution of blurred image can be done by experimentation, mathematical modeling and observation. Experimentation requires similar equipment used to acquire the degraded image, which is very rare in most real world cases. Majority of research is going on towards mathematical modeling to provide general solution. For very specific circumstances like restoring an old photograph of historical value, extracting important information like vehicle number from blurred image, estimation by image observation is a useful method. Experimental results show adequate restoration on unknown motion blurred images. Though this is very simple method, it is time consuming method for small volume. Work is going on to extract blur kernel from the blurred image using artificial neural network to reduce human efforts.

\section{ACKNOWLEDGMENTS}

This work was supported in part by the Physical Research Laboratory - Ahmedabad, (India) under PLANEX project (Planetary Exploration Technology Research).

\section{REFERENCES}

[1] J. Ko, and C. Kim, "Low Cost Blur Image Detection and Estimation for Mobile Devices", 11th International Conference on Advanced Communication Technology, South Korea, 15-18 February 2009, vol. 3, pp. 16051610.

[2] S. Shaojie, W. Qiong, and L. Guohui, "Image Restoration for Single Blurred Image", IEEE International Conference on Intelligent Computing and Intelligent Systems, Shanghai, 20-22 November 2009, vol. 4, pp. 491-495.

[3] A. Levin, "Blind Motion Deblurring Using Image Statistics", Neural Information Processing Systems, 2006, [Online]. Available: http://people.csail.mit.edu/ alevin/papers/levin-deblurring-nips06.pdf. 
[4] Y. You, and M. Kaveh, "A regularization approach to joint blur identification and image restoration", IEEE Transactions on Image Processing, vol. 5, issue 3, pp. 416-428, March 1996

[5] Q. Shan, J. Jia, and A. Agarwala, "High-quality Motion Deblurring from a Single Image", ACM Transactions on Graphics, vol. 27, no. 3, pp. 73:1-73:10, August 2008.

[6] Z. Hu, J. Huang, and M. Yang, "Single Image Deblurring With Adaptive Dictionary Learning", Proceedings of 2010 IEEE 17th International Conference on Image Processing, Hong Kong, 26-29 September, 2010, pp. 1169-1172.

[7] W. Hu, J. Xue, and, N. Zheng, "PSF Estimation via Gradient Domain Correlation", IEEE Transactions On Image Processing, vol. 21, no. 1, pp. 386-392, January 2012.

[8] F. Krahmer, Y. Lin, B. McAdoo, K. Ott, J. Wang, D. Widemann, and B. Wohlberg, "Blind Image Deconvolution: Motion Blur Estimation", technical report for the Mathematical Modeling in Industry X Workshop, Institute for Mathematics and its Applications, Minneapolis, September, 2006, [Online]. Available: https://www.ima.umn.edu/preprints/sep2006 12133-5.pdf

[9] P. Subashini, M. Krishnaveni, and V. Singh, "Image Deblurring Using Back Propagation Neural Network", World of Computer Science and Information Technology Journal (WCSIT), vol. 1, no. 6, 2011, pp. 277-282, 2011.

[10] I. Aizenberg, C. Butakoff, V. Karnaukhov, N. Merzlyakov and O. Milukova, "Blurred Image Restoration using the Type of Blur and Blur Parameter Identification on the Neural Network", proceedings of SPIE, Vol. 4667, May, 2002.

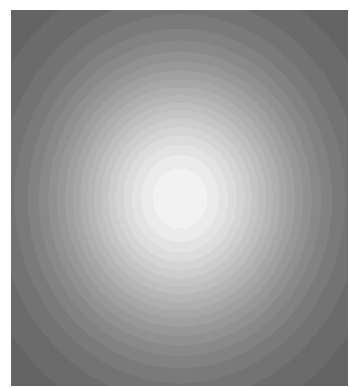

(a)

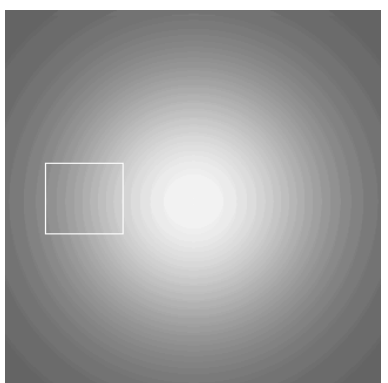

(b)

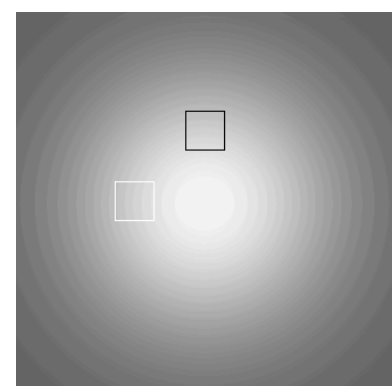

(c)

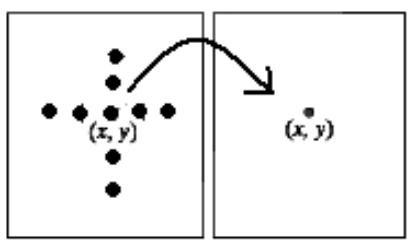

Latent image Translated image

Fig. 1. Value of each pixel in translated image is calculated as per central difference interpolation where central pixel location is ceiling value of interactively selected blur kernel.

Fig. 2. (a) Original image obtained from Gaussian function, (b) effect on blurred image when blur and gradient are orthogonal, (c) maximum gradient of horizontal and vertical segment of blurred image, (d) horizontal segment, (e) vertical segment. 

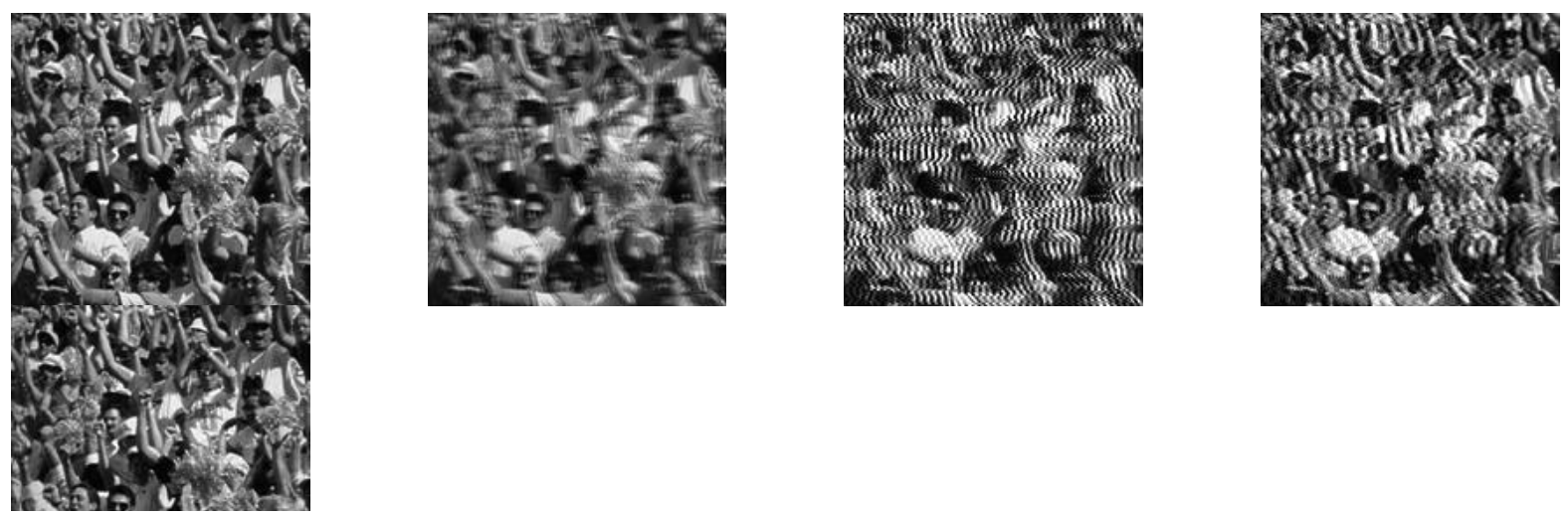

\subsection{Rotating ND array}

When sotucing an $\mathrm{ND}$ ams $\mathbf{I}$ we perficrmed. The peremil case is to no by dima and dim2. In the cases abe a pline spuened by dirpensions coe ifbeth size (I, dima) and size

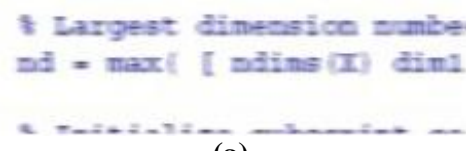

(a)

\subsection{Rotating ND array}

When cotating an ND array $\mathrm{X}$ we $\mathrm{t}$ performed. The general case is to ro by diml and dim 2 . In the cases abc a plane spanned by dimensions one i if both size ( $\mathrm{X}$, diml) and size

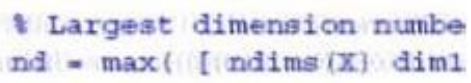

\subsection{Rotating ND array}

When rotating an $\mathrm{ND}$ array $\mathrm{X}$ we $\mathrm{I}$ performed. The general case is to ro by dim 1 and dim 2 . In the cases abc a plane spanned by dimensions one ( if both size ( $\mathrm{X}, \mathrm{dim} 2)$ and size

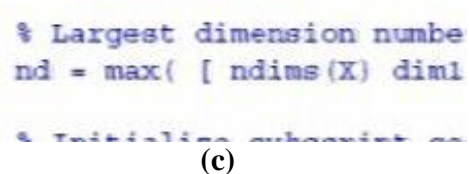
(c)

Fig. 4. Restoration of blurred image (a) blurred image obtained from [1], (b) result of the Ko. et al. [2009] in which blur is effectively present on the left side (c) our result.

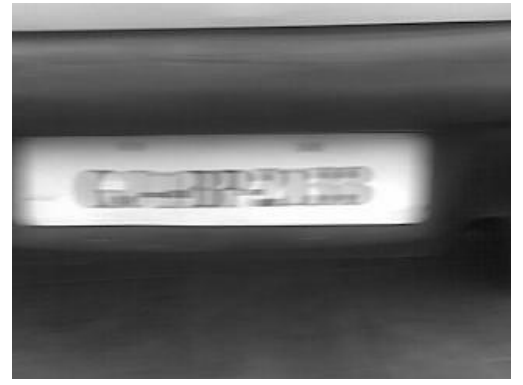

(a)

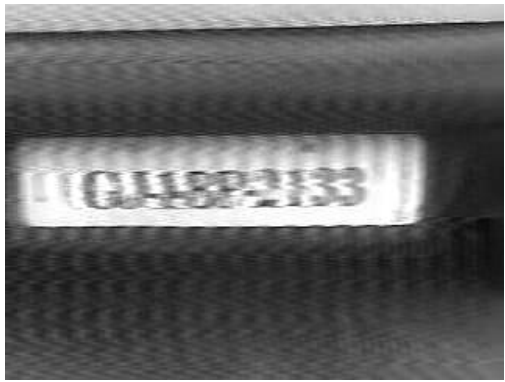

(b)

Fig. 5. Restoration of captured blurred image (a) blurred number plate "GJ-1 BP-2133"captured from in motion mobile phone camera of 2 megapixel spatial resolution, (b) our result. 